\title{
GÉNERO Y EDUCACIÓN: LAS ALTERNATIVAS AL FEMINISMO LIBERAL
}

\author{
MARTA JIMÉNEZ JAÉN \\ Universidad de La Laguna
}

\begin{abstract}
A partir de la articulación de las políticas de igualdad de oportunidades en la educación para las mujeres ha sido posible constatar la existencia de importantes limitaciones difícilmente resolubles y explicables en los términos establecidos por el denominado "feminismo liberal». En la reflexión crítica sobre esta perspectiva han aparecido aportaciones que, como ya constatamos en otro trabajo ${ }^{1}$, son herederas de los análisis de la educación vertidos en el marco de las sociologías críticas con el funcionalismo. La línea divisoria fundamental entre estos enfoques críticos es la que se puede establecer entre las críticas estructuralistas (inspiradas básicamente en las teorías marxistas y culturalistas de la reproducción) y los enfoques postestructurales (inspirados, entre otras, en las elaboraciones de M. Foucault y el marxismo culturalista).

En este trabajo vamos a exponer las principales aportaciones de estos enfoques que redundan en una idea central: la crítica de la pretendida neutralidad de las instituciones educativas en términos de las relaciones de género.
\end{abstract}

\section{LA TEORÍA DE LA REPRODUCCIÓN DEL SEXISMO}

El debate sobre el proceso reproductor de la división sexual de trabajo ha sido limitado. La idea central es, básicamente, la imposibilidad de que en una sociedad como la actual (patriarcal y capitalista) se llegue a erradicar la discriminación. Esta consideración remite, entonces, a una crítica radical de la sociedad $y$, en consecuencia, del optimismo respecto a las posibilidades del cambio a través de la educación: es incongruente esperar del sistema educativo en esta sociedad un efecto beneficioso para las mujeres. En este sentido, comparten, con matizaciones, la idea de que el sistema educativo se caracteriza eminentemente por su función «reproductora» en la sociedad. En Sociología de la Educación

1. Ver al respecto: JIMÉNEZ JAÉN, M.: «Género y educación: las aportaciones del feminismo liberal», Témpora, Vol. 3, $2^{a}$ época (2000). 
vienen a coincidir con los «teóricos de la reproducción», tanto en sus vertientes «culturalistas» como «economicistas».

Se da importancia al intento demostrar el rol de la enseñanza en la reproducción de una fuerza de trabajo femenina, bien para realizar un trabajo asalariado subalterno, bien para la realización del trabajo doméstico que proporciona al capital la crianza de las nuevas generaciones de trabajadores y que asegura a diario, mediante su servicio gratuito, la reproducción de la propia fuerza de trabajo ${ }^{2}$.

El feminismo socialista ha concedido especial importancia a poner de manifiesto que la igualdad de oportunidades en la escuela capitalista no sólo no erradica, sino que mantiene y legitima los fundamentos de la división sexual del trabajo en estrecha relación con la división capitalista del trabajo y las relaciones de explotación y subordinación entre las clases sociales.

Muchos de estos análisis están realizados bajo la influencia de las elaboraciones de M. Barrett, que intentó extrapolar las tesis althusserianas de la «autonomía relativa» del Estado a la problemática de la ideología del patriarcado. La dificultad más obvia para ello estriba en que Althusser se ocupó de la reproducción de las ideologías de clase, no de las de género, y existen problemas para considerar fructífera la extrapolación de sus tesis al género.

Barrett se enfrenta a la cuestión de por qué las mujeres están oprimidas, cuáles son las razones de su opresión y los términos en que están establecidos los conceptos de masculinidad, feminidad y práctica sexual, la ideología y la producción cultural del género, el sistema educativo, la división del trabajo, la familia, el estado y el capitalismo y la liberación de la mujer. Junto a la cuestión de la opresión de las mujeres, critica muchas elaboraciones sociológicas previas sobre las instituciones británicas -el estado, la familia, el sistema educativo, las relaciones industriales.$-{ }^{3}$ La respuesta, para la autora, se encuentra en la investigación histórica, en establecer la historia de la ideología de género en relación con el desarrollo de las relaciones sociales capitalistas.

Un concepto al que ella da importancia es el de "articulación», para defender la crucial importancia de la ideología en la construcción de sujetos con género y prácticas y políticas sociales con género. Cuando se analizan las «desigualdades» de género en el presente y las necesidades de la producción capitalista, la historia, y en particular la historia de la ideología de género, aporta el vínculo necesario:

«Se ha comprobado que el capitalismo no podría sobrevivir sin la forma presente del trabajo doméstico. Por otro lado es igualmente difícil identificar el desarrollo de la familia sin su relación con las necesidades de la producción capitalista. Las evidencias históricas avalables sugieren que no es adecuada ninguna generalización. Podría ser más útil considerar las formas en que las divisiones de género pre-capitalistas

2. ARNOT, M.: "La hegemonía masculina, las clases sociales y la educación de la mujer», Témpora, $n^{\circ}$ 9, Enero-Junio (1987), p. 73. 
han sido incorporadas, posiblemente exageradas, en la estructura de las relaciones capitalistas de producción» ${ }^{4}$

En la respuesta al dilema de la relación capitalismo/ideología patriarcal la autora es cautelosa:

«Es, quizás, posible resolver este problema sin recurrir a la tesis absolutamente paralizante de la «autonomía absoluta», o a una forma de materialismo que desplaza la contradicción capital/trabajo de su centralidad en el análisis de la sociedad capitalista. Primero, podemos afirmar que la ideología de género -el significado de la masculinidad y la feminidad- ha variado históricamente y no debería ser percibida como estática o unificada, por lo que debería analizarse en diferentes contextos históricos y de clase en los que tiene lugar. Segundo, podemos afirmar que el significado del género en el capitalismo actual se apoya en una estructura y división del trabajo doméstico que ocupa un lugar particular en las relaciones de producción, y que, además, esta ideología tiene, concretamente e históricamente, alguna base material. Tercero, podemos reconocer la dificultad de establecer categorías económicas e ideológicas como exclusivas y distintas. Las relaciones de producción y reproducción del capitalismo contemporáneo pueden operar en general de acuerdo a procesos de acumulación de capital explotadoras que son técnicamente neutrales, pero toman la forma de una división del trabajo que está sutilmente incorporada» ${ }^{5}$

Así, la autora consideró que la necesidad de acceder a una interpretación materialista de la ideología patriarcal requería de una concepción de ésta en los términos de Althusser: una visión de esta ideología separada de las relaciones capitalistas de producción sería idealista, pero tampoco sería congruente percibirla en absoluta dependencia de la explotación económica. La insistencia de Althusser en que los AIEs son «relativamente autónomos» permite concebir las cuestiones de género y las prácticas y políticas patriarcales en su particular especificidad, autónomas, pero no sin relación, con las relaciones de clase.

Este tipo de supuestos inspiraron el trabajo orientado a evidenciar cómo la escuela reproduce las relaciones sociales patriarcales. Los primeros esfuerzos en ese sentido fueron desplegados por A. Wolpe y R. Deem en Inglaterra. Un aspecto central de sus análisis fue contemplar las implicaciones económicas y sociales de los sistemas educativos, evidenciando su parcialidad.

Por un lado, A.M. Wolpe, a finales de los setenta, inició una línea de trabajo de cuestionamiento de los informes oficiales sobre la escolarización en Inglaterra, inspirados en la tesis del rol sexual ${ }^{6}$. La autora consideraba en este texto que los informes centrados exclusivamente en los problemas del acceso, las elecciones, las desventajas de las mujeres o su inferioridad numérica conciben sus dificultades en términos de diferencias psicológicas individuales y

3. LANDRY, D. - MACLEAN, G.: Materialists Feminisms, Oxford, Backwell, 1993, p. 28.

4. Ibídem, p. 29.

5. Citado en ibídem, p. 30.

6. "The official ideology of education for girls», en FLUDE, M. y AHIER, J. (eds.): Educability, schools and ideology, Londres, Croom Helm (1976). 
que, por tanto, son resolubles con medidas concretas en la orientación de sus expectativas. Para ella, esas interpretaciones ocultan el hecho de que la propia escuela no es neutral, que "las fuerzas potentes de la economía capitalista", con su necesidad de trabajo doméstico gratuito y de reserva de fuerza de trabajo, presiden la organización misma del sistema educativo ${ }^{7}$. La realidad del trabajo doméstico y asalariado de las mujeres se oculta en los informes oficiales y de esta manera han contribuido a la construcción de la ideología sustentadora de la opresión de género.

En un sentido similar, R. Deem en diversos trabajos ${ }^{8}$, analiza los datos sobre la escolarización de las niñas y jóvenes y sobre el trabajo femenino, defendiendo que las escuelas se muestran como instituciones centrales para el mantenimiento y la reproducción de la división sexual del trabajo: la escolarización juega un papel crucial en la formación de las mujeres para cierto tipo de trabajos a través de la transmisión de unos contenidos con sesgo de género y de la escasa presencia de mujeres en ciertas asignaturas y ámbitos de especialización (tecnologías, ciencias, matemáticas). La escuela no crea la división sexual del trabajo, pero no hace nada para atacarla; al contrario, transmite culturas diferentes a los niños y niñas de modo que, cuando éstos realizan elecciones, lo hacen reflejando la división sexual del trabajo.

Estas autoras, en definitiva, ejemplifican un tipo de análisis que deriva de la aplicación al problema del sexismo del modelo de análisis derivado de las teorías de la reproducción, reflejando a su vez los principales límites de las mismas y, en particular, tal como resaltan entre otras K. Weiler y M. Arnot:

- El uso parcial del concepto de ideología, entendida como «la imposición incontestada de una visión de la realidad o de un conjunto de valores».

- La centralidad que asignan a la problemática relación entre escolarización y trabajo femenino, no dando pie a la consideración de otro tipo de cuestiones vinculadas al género como la sexualidad.

- No evidenciar los procesos y prácticas cotidianos a través de los cuales operan las relaciones de género en las escuelas impide constatar la existencia de contradicciones, tensiones y conflictos en el proceso mismo de la reproducción de la división sexual del trabajo, obviando las resistencias protagonizadas por las mujeres en el sistema educativo, tanto las enseñantes como las alumnas?.

El énfasis puesto por las primeras versiones del feminismo marxista en dar cuenta de la funcionalidad material e ideológica -aunque fuera en última instancia- del patriarcado para el capitalismo, así como la incondicional vinculación que el feminismo socialista establecía entre la lucha de las mujeres y la lucha de

7. WEILER, K.: Women teaching for change. Gender, class and power, Massachussets, Bergin and Garvey Publishers, 1988, p. 36.

8. Por ejemplo, R. DEEM: Schooling for women's work, Londres, Routledge and Kegan Paul, 1980.

9. WEILER, K.: opus cit, pp. 33 y ss. También, ver ARNOT, M.: «La hegemonía masculina,...», opus cit., p. 73. 
clases, enfatizando como objetivo a su vez la obtención de la igualdad (ahora real) con los hombres pero desde la liberación de la clase obrera, además, favoreció las críticas desde el «feminismo radical» cuestionando el determinismo económico como marco explicativo. Esta orientación del feminismo enfatiza la responsabilidad central de los hombres -no del capital- en el surgimiento y mantenimiento de la situación de subordinación de las mujeres, en tanto que directos beneficiarios de la misma. Estas tesis, por tanto, destacan la centralidad del poder individual y colectivo de los hombres, llegando a asumir que la historia del patriarcado ha dado lugar efectivamente a dos culturas irreconciliables que sustentan modos de vida e intereses enfrentados entre los sexos. Las vertientes más radicales llegan a defender la indeseabilidad de las aspiraciones igualitarias respecto a los hombres:

"La igualdad en este contexto puede suponer solo la abstracta oportunidad de volverse igual a los hombres. Es el cuerpo masculino, y sus poderes y capacidades determinados histórica y culturalmente, lo que se asume como norma del individuo liberal. Las mujeres pueden lograr este estándar siempre que o eludan su propia especificidad corporal, o sean capaces de hacer malabarismos entre su rol tradicional en la esfera privada y su nueva igualdad. Esta situación oculta los poderes y capacidades que las mujeres han desarrollado en su contexto histórico y cultural.» ${ }^{10}$

En estas tesis radicales, el concepto de "reproducción» es bien diferente del término marxista, y remite básicamente a la consideración de que las dinámicas y espacios culturales en la sociedad no necesariamente están vinculados a los intereses económicos y políticos de las clases dominantes. El sexismo, los espacios y mecanismos a través de los cuales se genera y reproduce, se muestra como un proceso eminentemente cultural que es preciso desentrañar en su peculiar complejidad, sin necesariamente remitir a las dinámicas de la lucha de clases ni a los requerimientos económicos de la sociedad capitalista: "la meta de la educación feminista, escribe M. O'Brien, no es la igualdad en el conocimiento, el poder y la riqueza, sino la abolición del género como realidad cultural opresiva». ${ }^{11}$

A partir de estas críticas, se han desplegado numerosas investigaciones sobre el sexismo en la escuela que han tratado de problematizar el monopolio de la cultura por parte de los hombres, de un lado, y de otro los procesos internos a través de los cuales se procede a la formación de identidades diferenciadas en ambos géneros en las escuelas. En particular, han proliferado los estudios sobre el «fracaso» escolar, el currículo oculto y manifiesto, las interacciones que se dan en el aula, las actitudes de las niñas ante la escuela, así como las actitudes del profesorado y los orientadores vocacionales y educativos ante el futuro de las niñas... Los estudios "culturalistas» intentan, en definitiva, desmitificar los

10. AMORÓS, C.: «La ideología de la división sexual del trabajo», En Teoría, nº 2 (Jul-Sept), (1979), p. 127. En un sentido similar: ARNOT, M.: «Feminismo y democracia», en Varios Autores: Volver a pensar la educación (Vol. I), Madrid, Morata/Paideia (1995), pp. 307 y ss.

11. ACKER, S.: Género y educación, Madrid, Narcea, 1995, p. 72. 
contenidos y las prácticas que se despliegan cotidianamente en las escuelas, pero sin necesariamente remitir a las dimensiones «estructurales» de los sistemas educativos. Ejemplos de estos desarrollos son las obras de D. Spender sobre el sesgo masculino de los contenidos de la enseñanza, las posiciones de dominio de los hombres en los contextos educacionales y la función del lenguaje en el control de las formas en que las mujeres se conceptualizan a sí mismas ${ }^{12}$.

La denuncia del conocimiento y la interacción escolar se dirige en un doble sentido: en un primer momento, predominan los estudios que tratan de mostrar que los libros de texto y los currículos escolares emiten mensajes que tienden a reforzar el rol tradicional de las mujeres y, por otro lado, se denuncian los mecanismos de participación en la vida educativa que dificultan el acceso de las mujeres a puestos de responsabilidad en los centros escolares y en el sistema educativo en general ${ }^{13}$. En una segunda fase, en que se intentan integrar ciertos aportes del feminismo radical sobre la positividad de los «valores femeninos», se resalta, al contrario, la exclusión de estos valores y experiencias en el conocimiento escolar, así como la infravaloración de "lo femenino» en las relaciones sociales escolares (en el lenguaje, las interacciones profesorado/alumnado, etc. $)^{14}$

A partir de los años ochenta, tal como recoge S. Acker, también se ha iniciado una línea de trabajo que insiste en cómo los chicos varones (y también a veces los profesores) reproducen las conductas violentas en las escuelas acosando sexualmente, degradando y avergonzando a las chicas (y en ocasiones a las profesoras) ${ }^{15}$.

Los diversos trabajos centrados en la denuncia de la parcialidad de las instituciones y procesos educativos sin duda alguna han jugado un papel crucial, tanto en el desarrollo del pensamiento sociológico como del propio movimiento teórico, político y educativo feminista: nunca más las visiones ingenuas de la educación se han podido sostener en el marco de las investigaciones, aunque ello no haya impedido que desde los discursos educativos oficiales se siga mostrando el problema de las mujeres como estrictamente una cuestión de «oportunidades». Sin embargo, no se puede dar por cerrado el debate sobre la discriminación sexista con estos avances. De hecho, frente a los análisis «reproduccionistas», se han vertido numerosas críticas, que apuntan precisamente a las derivaciones mecanicistas que los han presidido: la idea de que la enseñanza sea concebida como un mero espacio «reproductor» de lo que ocurre en la sociedad sin que se contemplen, bien su carácter contradictorio, bien su carácter productivo de identidades sociales más allá de lo que viene establecido por las dinámicas sociales. Las alternativas teóricas, en definitiva, se sitúan en estrecha relación con los desarrollos post-estructuralistas tanto de la Sociología de la

12. Citado por ACKER, S.: ibídem, pp. 72-73.

13. BALL, S.J.- La micropolítica de la escuela, Barcelona, Paidós/MEC, 1989.

14. Por ejemplo, M. SUBIRATS y C. BRULLET: Rosa y Azul. La transmisión de los géneros en la escuela mixta, Madrid, Instituto de la Mujer, 1988.

15. ACKER, S.: Género..., opus cit., p. 73. 
Educación como del propio feminismo, si bien es preciso reconocer que el sentido teórico de estas críticas se sustenta, una vez más, en visiones contrapuestas de la educación y la sociedad.

\section{ANÁLISIS POST-ESTRUCTURALISTAS: LA «CONSTRUCCIÓN SOCIAL DEL GÉNERO»}

La preeminencia de los planteamientos estructuralistas en el seno de la Sociología de la Educación empezó a ponerse en crisis a lo largo de los años 80 , en que la "nueva sociología de la educación», bajo la influencia de ciertas perspectivas inspiradas en las elaboraciones de Max Weber y del denominado "interaccionismo simbólico», tratan de rescatar para la investigación sociológica la importancia de los agentes sociales. También en el seno de la tradición marxista el modelo althusseriano es sometido a un extenso debate desde diversas vertientes que intentan rescatar de la obra de Marx los elementos más vinculados a la subjetividad. En el marco del feminismo, por último, se ha dejado sentir el influjo de algunas elaboraciones denominadas «post-modernistas» que, como veremos, parten de una crítica a los fundamentos epistemológicos de las "teorías modernas» y su confianza en los principios universales, su búsqueda de relaciones causales en los procesos sociales y su apoyo a la racionalidad como principio estructurador del pensamiento y las prácticas sociales ${ }^{16}$.

Dada la diversidad enorme de análisis y teorías que se han empezado a desarrollar en los últimos años, se nos hace imposible realizar una síntesis que nos permita tener una visión panorámica de los principales aportes. Nos centraremos, más bien, en dos líneas de investigación que, a nuestro modo de ver, se han mostrado con particular interés de cara a la comprensión de las dinámicas de la imposición y las resistencias al poder de género en el ámbito educativo: el "marxismo culturalista» ${ }^{17}$ y las tesis de la "genealogía del poder» inspiradas en las elaboraciones de $\mathrm{M}$. Foucault ${ }^{18}$.

\subsection{Foucault y los análisis del «discurso»: el conocimiento escolar como "productor" de categorías sexuales}

Las críticas más rotundas a los planteamientos «estructuralistas» provienen de corrientes de pensamiento no estrictamente sociológicas. Desde diversas elaboraciones filosófico-epistemológicas se afronta, en los años ochenta, una crí-

16. Ver, por ejemplo: BARRETT, M.: «Words and Things: Materialism and Method in Contemporary Feminist Analysis», en BARRETT, M.-PHILLIPS, A. (Eds.): Destabilizing Theory. Contemporary Feminist Debats, Cambridge, Polity Press, 1992, pp.201 y ss.

17. En particular, las elaboraciones sobre el Estado y la cultura que diversos autores han desarrollado a partir de las tesis gramscianas sobre los procesos de hegemonía. Ver, al respecto, por ejemplo: WILLIAMS, R.: Cultura, Barcelona, Paidós, 1982; CABRERA, B.: «Culturas sociales y culturas escolares en la Sociología de la Educación", Témpora, n 17-18, 1991; MORGENSTERN, S.: "Antonio Gramsci: hegemonía y educación», en GÓMEZ R. DE CASTRO, F. et al: Socialismo y sistemas educativos, Madrid, UNED, 1991.

18. Como obras de mayor relevancia en este tipo de debates, destacan de este autor: Vigilar y castigar, Madrid, Siglo XXI, 1988; Historia de la sexualidad (varios tomos), Madrid, Siglo XXI, 1989. 
tica a los fundamentos de la denominada "Gran Teoría»y, particularmente, se renuncia al intento de ofrecer una «explicación» genérica sobre los fundamentos de la sociedad. Las elaboraciones de M. Foucault tienen especial incidencia en la Sociología en la medida en que se ocupan particularmente de ofrecer caracterizaciones alternativas de las dinámicas del poder en la sociedad. En el terreno de la sexualidad y de la educación, este autor ha desplegado trabajos de sumo interés que han sido asumidos -aunque no siempre haciendo justicia a su proyecto político e intelectual- por no pocas estudiosas feministas y sociólogos/ as de la educación ${ }^{19}$. En Foucault una principal fuente de preocupación es el análisis del poder, pero no entendido en términos de "estructuras», ni de una «propiedad» que es poseída por ciertos grupos sociales y vertida sobre otros: para él el poder es, principalmente, una relación que permea la totalidad de la vida social, y es ejercido en todas las instancias donde los individuos entablan relaciones: la sexualidad, el trabajo, el estudio, la enfermedad,... Frente a las consideraciones que conciben la existencia de relaciones de poder que vienen dadas más allá de la subjetividad de los individuos, propugna la idea de que todos los agentes sociales participan activamente de relaciones de poder: son a la vez agentes del poder y objetos del mismo. El análisis social debe contemplar, entonces, no tanto el problema de las intencionalidades de los grupos que «poseen» el poder, como las técnicas y estrategias a través de las que el poder atraviesa el conjunto de las relaciones entre los seres sociales. El poder no es una consecuencia de una estructuración dada de la sociedad, sino que es una relación productora de realidades y relaciones sociales: para Foucault son los «discursos», y no las "estructuras sociales», los que dan lugar a realidades sociales; los "discursos» son, en realidad, conjunciones de formas específicas de saber y de poder que no actúan simplemente de forma "represiva», sino que son "productivos»: nuestras concepciones de la realidad y de nosotros mismos están conformadas a partir de ciertas reglas que determinan lo que puede decirse y lo que puede pensarse o sentirse en un "discurso" concreto y en un tiempo determinado. No existen, por tanto, categorías prefijadas de la realidad, sino discursos diferentes a lo largo de la historia, de forma que cuando utilizamos términos como el de "mujer", no nos estamos refiriendo a una realidad objetiva, a una condición que mantiene a lo largo de la historia una continuidad, sino a una multiplicidad de discursos que se han construido socialmente a lo largo del tiempo.

Este tipo de planteamientos tiene una doble utilidad para el pensamiento feminista: por un lado, las elaboraciones de Foucault sobre cómo actúa el poder a través del saber "produciendo» realidades y categorías sociales resulta de enorme interés para un colectivo social que ha visto su vida y sus experiencias excluidas o tergiversadas por el conocimiento; sus análisis sobre los procedimientos de prohibición, exclusión, oposición entre verdadero/falso del conocimiento «dis-

19. Ver, por ejemplo: BALL, S. (ed.): Foucault y la educación. Disciplinas y saber, Madrid, Morata, 1993. 
ciplinario", así como los procedimientos de clasificación, categorización, distribución, etc., de los individuos, resultan de extrema importancia aquí. Por otro lado, las consideraciones de este autor sobre la permeabilidad del poder abren la puerta para dejar a un lado la necesidad de demostrar la sustantividad de las relaciones entre los géneros como problema sociológico, al tiempo que favorece que los estudios abandonen la discusión en términos abstractos para pasar a centrarse en el análisis de "las condiciones históricas y sociales de la aparición de los discursos y su relación con los poderes institucionales» ${ }^{20}$. Asimismo, la crítica del conocimiento escolar se ve favorecida desde que el eje saber/poder es posible ponerlo de manifiesto sin tener que previamente establecer un marco «estructural» de relaciones entre la subordinación de las mujeres y la de otros grupos sociales: el saber no se considera neutro, puro, o verdadero o falso en sí mismo, sino que forma parte de «regímenes de verdad» diversos histórica y políticamente. Por otro lado, la problemática de las mujeres puede ser comprendida específicamente y remitirse a cada contexto y momento particular, de modo que también se puede llegar a abarcar algo que había sido desestimado por las teorías feministas estructuralistas: las diferencias entre las mismas mujeres, producto de las «prácticas divisorias» ejercidas desde el saber.

La conclusión a la que se puede llegar frente a las tesis de la reproducción es, sin embargo, controvertida: estos análisis permiten romper con las versiones de lo educativo como un ámbito de la realidad predeterminado, y permiten acometer líneas de investigación donde es más importante la caracterización de los procesos de "construcción social» de las relaciones de poder incorporadas al conocimiento educativo y a las vivencias cotidianas de las escuelas. Sin embargo, este modelo analítico presenta, a nuestro modo de ver, problemas no resueltos: de un lado, la negación de toda posibilidad de que el conocimiento en sí pueda constituir, a pesar de sus implicaciones políticas, un instrumento de comprensión crítica de la realidad, imprescindible para la incardinación de proyectos transformadores; $y$, de otro, la imposibilidad de admitir la existencia de una jerarquización entre las distintas formas y relaciones de poder que se contemplan en la sociedad. En este terreno, si bien es asumible la constatación de dimensiones políticas en todos los niveles de las relaciones entre seres humanos, no obstante no resulta demasiado clarificador proceder a una equiparación plena entre distintas manifestaciones y vivencias del poder, pudiéndose caer efectivamente en un nuevo modo de concebir ingenuamente la posibilidad de los cambios: si ninguna instancia se identifica como central, tiene la misma relevancia el compromiso en cualquier espacio. Las tesis de Foucault resultan «liberadoras» de muchos de los esquematismos y cerrazones del estructuralismo, pero tampoco llegan a resolver muchos de sus dilemas. Precisamente donde estos planteamientos son débiles es posible recurrir a otros marcos analíticos

20. KENWAY, J.: «La educación y el discurso político de la Nueva Derecha», en BALL, S. (ed.): Foucault y la educación. Disciplinas y saber, opus cit., p. 175. 
que, no obstante, también permiten concebir de forma compleja la realidad social y educativa.

\subsection{Desarrollando una teoría de la "hegemonía masculina"}

A partir de las elaboraciones de Gramsci sobre la «hegemonía» en las sociedades capitalistas, también es posible cuestionar las concepciones deterministas que consideran la escuela y la cultura como partes de la "superestructura» que, a su vez, operan mecánicamente sometidas a los designios de la «infraestructura económica». La noción gramsciana de "hegemonía» permite concebir las relaciones de dominio y subordinación de forma compleja, situando la lucha de clases en su origen y mantenimiento. La hegemonía refiere a toda una serie de estructuras y de actividades, así como de valores, actitudes, creencias, moralidad,... que apoyan, de manera compleja y diversa, el orden establecido y los intereses de los grupos "hegemónicos» en la sociedad. La hegemonía tiene, en sí, un carácter político, pero efectivamente abarca las dimensiones culturales de las relaciones sociales: para Gramsci, un aspecto central de la hegemonía es, precisamente, el consentimiento activo por parte de los grupos subordinados de las normas y valores establecidos. El dominio político es también cultural en la medida en que, más allá de las normas, valores y significados institucionalizados, abarca los estilos de vida, las expectativas y experiencias vitales de los individuos y grupos sociales subordinados y, en este terreno, no siempre es efectiva la simple imposición por parte de los sectores hegemónicos: se requieren niveles importantes de identificación activa de los seres sociales con los discursos y prácticas hegemónicos.

Esta teorización pone en primer término, entonces, la importancia de la actividad de los agentes sometidos a la hegemonía, en un doble sentido: si se requiere su identificación, los discursos hegemónicos de forma compleja habrán de contener elementos culturales no estrictamente genuinos de los grupos hegemónicos; habrán, por tanto, de ser continuamente renovados... porque continuamente se pueden ver desafiados. Por otro lado, la noción de hegemonía remite a la posibilidad histórica de la contrahegemonía, de proyectos sociales, culturales y políticos emanados de los grupos subalternos que remiten a una transformación global de la sociedad.

Un sistema cultural dominante, una determinada visión de la vida, nunca, como afirmaba Foucault, es estable ni definitivo; se ve sometido a numerosas transformaciones y contradicciones que se gestan a partir de la lucha entre los grupos sociales (las clases sociales y/o sectores de las mismas) que participan de intereses y experiencias diferenciados y/o enfrentados.

Según M. Arnot ${ }^{21}$, si bien Gramsci desarrolló esta teorización pensando exclusivamente en las relaciones de hegemonía entre las clases sociales, es necesario integrar en este marco analítico las relaciones entre los géneros, al tiempo que utilizar los elementos básicos del concepto de «relaciones hegemó-

21. "La hegemonía masculina,...», opus cit., pp. 67 y ss. 
nicas» para aproximarnos a una comprensión no mecanicista de las relaciones de poder entre hombres y mujeres: para ella, es posible concebir la existencia de una "hegemonía masculina» en las sociedades capitalistas, entendiendo por ello "...un conjunto de 'circunstancias' diversas a través de las cuales las mujeres han ido aceptando una cultura dominada por el hombre, su legalidad y su subordinación a ambas»; las «circunstancias educativas» constituirían, así, "...un modelo de experiencia femenina cualitativamente diferente a la masculina» ${ }^{22}$, que adopta formas y contenidos diferentes según las clases y sectores de clases sociales. El aprendizaje, así, es entendido como un proceso que, sin permanecer al margen de las dinámicas y relaciones sociales de poder, tiene una naturaleza activa, estando atravesado por relaciones dialécticas, luchas de poder y conflictos donde pueden jugar un papel relevante las distintas alternativas prácticas que pueden existir dentro y fuera de la escuela. No obstante, la posibilidad de que existan proyectos contrahegemónicos no asegura, en sí, su éxito: éste dependerá de la situación de la lucha (de clases, de géneros) y de la capacidad organizativa que en cada momento adquieran los grupos sociales enfrentados en el conjunto de la realidad social.

Para esta autora, tres características centrales hay que tener presentes en tanto que premisas a la hora de acometer la investigación sobre cómo y por qué se construyen los modelos de género de la manera en que se está haciendo en nuestra sociedad:

1) Las categorías sexuales son "constructos sociales arbitrarios» cuya forma y contenidos concretos son producto de la actividad de una gran diversidad de instituciones y agentes sociales.

2) Las clasificaciones de los sexos no son ni universales, ni simples, ni estáticas; son altamente complejas en sí mismas y existe tanta tensión en el interior de cada modelo como entre ellos.

3) Las clasificaciones sexuales se han construido en el contexto de las relaciones entre clases y entre géneros, no pudiéndose realizar análisis sin abarcar las diferentes interacciones que confluyen en las experiencias y la vida de cada agente social.

Como se puede observar, existen puntos de encuentro con los planteamientos post-modernistas al enfatizar las diferencias, el carácter histórico y arbitrario de la codificación de género, pero se contempla, no obstante, que las identidades culturales son, a su vez, sociales: las condiciones y situaciones sociales que viven los sujetos y los grupos sociales inciden, de manera compleja y contradictoria, sobre su identidad, de modo que es preciso integrar en los análisis la consideración de las dimensiones colectivas que, a pesar de las diferencias, unifican a los miembros de un determinado grupo, al tiempo que no se renuncia al intento de insertar los análisis sobre la "construcción social de los géneros» en relación con el conjunto de circunstancias y dinámicas políticas, económicas y sociales que establecen límites e intervienen en las relaciones «microsociales» 
en espacios concretos como, por ejemplo, la escuela. Sólo de esta manera, se considera en esta perspectiva, es posible no caer en algunos de los principales errores de las teorías postmodernistas: el relativismo cultural ("todo vale»), la disolución de la política y de la dominación y, finalmente, la ingenuidad que puede derivar de ambos de cara a la conformación de proyectos y prácticas transformadores.

Un desarrollo específico dentro de este enfoque gramsciano viene dado por los trabajos que, tomando como referencia el enfoque aportado por P. Willis sobre las resistencias de ciertos grupos de estudiantes a la escuela, inciden de forma particular en la producción de las culturas de género en las chicas de clase trabajadora, siendo centrales en este sentido las aportaciones de A. McRobbie. Esta autora, junto a otros como Connell123, defiende que las resistencias tienen significados diferentes para las niñas y los niños, y que los procesos de conflicto en la escuela sólo pueden ser comprendidos en relación tanto a la posición de clase como a la de género. Más aún, como afirma K. Weiler, "la educación puede tener diferentes significados para ellas que para los niños de igual clase social y raza. (...) Las niñas y mujeres con diferentes subjetividades de raza y clase tendrán diferentes experiencias en la escuela. Sus resistencias y sus «lecturas» de los mensajes ideológicos de la escuela diferirán en aspectos educativos específicos. Y por supuesto niñas de distintas clase y raza serán objeto de expectativas diversas por parte de enseñantes mujeres y hombres, blancos y negros, dependiendo de las propias visiones que tengan sobre lo que es propio de cada género. $\mathrm{Al}$ añadir las categorías de raza y clase a la de género, podemos empezar a desvelar la diversidad y complejidad de las experiencias de las niñas $y$ las mujeres en las escuelas. ${ }^{24}$

A. McRobbie ha destacado por sus críticas al trabajo de Willis y el posterior desarrollo de investigaciones con niñas anti-sociales, en las que pone de manifiesto que «el propio sexismo de los niños reproduce el rol de las niñas en la cultura de clase obrera como oprimidas y subordinadas. Aunque estas relaciones pueden ser en algún sentido un reflejo de la lógica del capitalismo, no son la ideología o las políticas del estado capitalista las que directamente presionan a las niñas de clase obrera, sino más bien el sexismo inmediato y opresivo de los niños de clase obrera. ${ }^{25}$ Esta autora ha aportado estudios etnográficos de las subculturas femeninas en grupos de niñas de 14-16 años de distintas clases sociales, contemplando tanto su experiencia privada de la sexualidad como su relación con el mundo público de la calle y el trabajo asalariado, encontrando que una forma peculiar de resistencia de estos colectivos pasa por la sexuali$\mathrm{dad}^{26}$.

23. CONNELL, R.W.: Gender and Power, California, Stanford University Press, 1987.

24. WEILER, K.: Women teaching..., opus cit., p.40.

25. Ibídem, pp. 40-41.

26. Citado por Weiler, ibídem, p. 42. 
Thomas encontró en un estudio similar que la contracultura de las niñas frente a la autoridad de la escuela vacilaba entre la defensa agresiva y una afirmación de la sexualidad y a veces la coquetería, particularmente ante los profesores hombres más jóvenes. La sexualidad, lo que la sociedad suele asignarles como su rasgo más característico, es usada agresivamente en un acto de autoafirmación que, a su vez curiosamente reproduce parte de los discursos establecidos: así como los chicos de Willis enfatizaban su masculinidad y rechazaban el trabajo intelectual, las chicas de origen obrero enfatizan la feminidad en un sentido tradicional y acaban aceptando la explotación en el ámbito doméstico y en los empleos marginales y mal retribuidos ${ }^{27}$.

Como aportación particularmente de interés de estos trabajos resaltamos, siguiendo a Weiler, el énfasis puesto en que una verdadera comprensión de los procesos de producción cultural y resistencias en la que se abarquen conjuntamente la clase, el género $y$, en su caso, la etnia, debe partir del análisis de los procesos, prácticas y relaciones que tienen lugar tanto en el ámbito de la vida pública (la escuela, el trabajo, la calle) como en el de la vida privada (la familia, el trabajo doméstico, la sexualidad). Lo público y lo privado están profundamente imbricados, y ambos conforman un mundo cultural completo. Puesto que los niños y niñas, hombres y mujeres, están asociados a formas muy rígidas de vivencias en uno u otro ámbito, elaboran respuestas culturales individuales y colectivas bastante diferentes, aunque al mismo tiempo pueden ser complementarias: sólo esforzándonos por captar ambas accederemos más fehacientemente a la realidad concebida como una totalidad ${ }^{28}$.

Como la limitación más señalada de estos estudios hemos de mencionar, finalmente, el concepto en sí de "resistencia» que se emplea, en el que resistir queda equiparado a protagonizar conductas antisociales. Autoras como Fuller y Anyon consideran que pueden observarse e interpretarse como otras formas de resistencia específica al sexismo y la asignación de roles tradicionales a las niñas y mujeres el aferrarse al aprendizaje escolar como modo de evadirse del futuro rol de madre-esposa preasignado socialmente a las mujeres. El concepto de resistencia pasa a ser concebido como un complejo proceso de acomodación y resistencia en la negociación de las relaciones sociales por parte de ciertos colectivos de mujeres.

Esto último enlaza con un aspecto de la conceptualización gramsciana que contiene también aportes de interés que será preciso desarrollar en el futuro. Para Gramsci, el "pensamiento elaborado», si bien está inmerso y actúa en el conjunto de las relaciones de hegemonía en la sociedad, no obstante es en sí contradictorio: al tiempo que contiene elementos claves para el mantenimiento y ejercicio de la hegemonía (y aquí podría coincidir con Foucault), también contiene claves para acceder a la comprensión de la realidad que son de crucial importancia para la construcción de proyectos críticos y transformadores de la

27. Ibídem, p. 43.

28. Ibídem, p. 45. 
sociedad. Esta consideración compleja del conocimiento como un proceso contradictorio da lugar a una reflexión sustancialmente distinta sobre su relación con el problema del sexismo y la educación. Desde los análisis reproduccionistas, e incluso desde la perspectiva foucaultiana, la formación intelectual sería concebida negativamente: en el primer caso, porque se supone que el conocimiento escolar reproduce los estereotipos sexistas, y en el segundo porque constituye un instrumento central de la estrategia del poder. Algunas vertientes del feminismo radical podrían incluso promover el rechazo a dicho pensamiento precisamente por su carácter intrínsecamente «masculino»...

La teorización gramsciana no da pie a pensar ingenuamente en las posibilidades de la educación y del conocimiento elaborado para transformar la realidad y, de hecho, propugna una visión dialéctica de las relaciones entre la actividad y la estructura, así como entre la lucha educativa y otras luchas. Pero, cuando menos, nos permite seguir pensando que la lucha no sólo es posible, sino que constituye un elemento estructurador de la realidad social y que una de las vertientes específicas de la lucha histórica de las mujeres ha tenido un sentido del que debemos congratularnos: la conquista del derecho a acceder a los instrumentos intelectuales de comprensión crítica del mundo. Muy probablemente, sin ellos los debates y el desarrollo de proyectos transformadores de nuestra vida no habrían llegado al punto en que nos encontramos.

\section{A MODO DE CONCLUSIÓN}

No cabe duda de que tampoco está asegurado el éxito del modelo de la hegemonía para servir de punto de partida para la investigación del sexismo. Las dificultades son importantes, pero pensamos en éste como un buen marco de análisis. También la incorporación flexible de las aportaciones de otras teorizaciones sobre las facetas desestimadas en el modelo inicial puede dar sus frutos si se empieza a trabajar en un sentido integrador. No obstante, todas estas consideraciones habrán de dotarse de una fundamentación sociohistórica, al mismo tiempo que deben considerar los procesos y dinámicas que efectivamente se ponen en marcha en cada contexto: sin ambas aportaciones será imposible el avance teórico y metodológico. No cabe duda de que el terreno está abonado para que las polémicas no desaparezcan a corto plazo, y quizás, en la medida en que nos referimos a realidades, significados, experiencias y circunstancias cambiantes, será imposible que algún día se llegue a pensar que todo está dicho. 